\title{
A helpful analogy between the covalent bond and Particle Spectroscopy
}

\author{
D. V. Bugg1 \\ Queen Mary, University of London, London E1 4NS, UK
}

\begin{abstract}
It is proposed that meson resonances are linear combinations of $q \bar{q}$ and meson-meson (MM); baryon resonances are combinations of $q q q$ and meson-baryon (MB). Mixing between these combinations arises via decays of confined states to meson-meson or meson-baryon. There is a precise analogy with the covalent bond in molecular physics; it helps to visualise what is happening physically. One eigenstate is lowered by the mixing; the other moves up and normally increases in width. Cusps arise at thresholds. At sharp thresholds due to S-wave 2-particle decays, these cusps play a conspicuous role in many sets of data. The overall pattern of light mesons is consistent with nearly linear Regge trajectories, hence $q \bar{q}$ components. There is no obvious reason why this pattern should arise from dynamically generated states without $q \bar{q}$ content.
\end{abstract}

PACS numbers: 12.39.Ki, 12.40.Yx, 13.25.Ft, 14.20.Gk

\section{Introduction}

In the early days of Particle Physics, Chew, Goldberger and others tried to account for resonances in terms of particle exchanges [1]. This met with partial success. For $\pi N$ elastic scattering, Donnachie and Hamilton [2] showed that exchanges of $N, \Delta(1232), \rho$ and $\sigma$ provide long range attraction in $P_{33}, D_{13}, D_{15}$ and $F_{15}$ partial waves where prominent resonances are observed. Furthermore, these exchanges account for repulsive partial waves $P_{13}, P_{31}, P_{11}, D_{33}$ and $D_{35}$. However, meson exchanges failed to account for the $\rho$ meson. This approach was therefore quickly overtaken by the quark model, which has provided a semi-quantitative picture of most of the known resonances.

However, there are cracks in this framework. Firstly, it does not account for the $\sigma$ and $\kappa$ poles, $a_{0}(980)$ and $f_{0}(980)$. Secondly, there are many examples where resonances appear at or close to sharp S-wave thresholds: e.g. $f_{0}(980)$ and $a_{0}(980)$ at the $K \bar{K}$ threshold, $f_{2}(1565)$ at the $\omega \omega$ threshold, $X(3872)$ at the $\bar{D}_{0} D_{0}^{*}$ threshold, $S_{11}(1650)$ and $D_{13}(1700)$ close to the $\omega N$ threshold.

There is a straightforward explanation of how S-wave thresholds attract these resonances [3]. Consider $f_{0}(980)$ as an example. Its amplitude for $\pi \pi \rightarrow K K$ is given to first approximation by the Flatté formula [4]:

$$
A_{12}=T_{12} \sqrt{\rho_{1} \rho_{2}}=\frac{G_{1} G_{2} \sqrt{\rho_{1} \rho_{2}}}{M^{2}-s-i\left[G_{1}^{2} \rho_{1}(s)+G_{2}^{2} \rho_{2}(s)\right]}=\frac{N(s)}{D(s)}
$$

where phase space $\rho$ is factored out of $T$. Here $G_{i}=g_{i} F_{i}(s)$ and $g_{i}$ are coupling constants, $F_{i}$ are form factors. Writing $D(s)=M^{2}-s-i \Pi(s)$, a more exact form for $D(s)$ is $M^{2}-s-$ $\operatorname{Re} \Pi(s)-i \Pi(s)$, where

$$
R e \Pi_{K K}(s)=\frac{1}{\pi} \mathrm{P} \int_{4 \mathrm{~m}_{\mathrm{K}}^{2}}^{\infty} \mathrm{ds}^{\prime} \frac{\mathrm{G}_{\mathrm{KK}}^{2}\left(\mathrm{~s}^{\prime}\right) \rho_{\mathrm{KK}}\left(\mathrm{s}^{\prime}\right)}{\left(\mathrm{s}^{\prime}-\mathrm{s}\right)} .
$$

\footnotetext{
${ }^{1}$ email: d.bugg@rl.ac.uk
} 
Fig. 1(a) illustrates the dispersive term $\operatorname{Re} \Pi(s)$ for $f_{0}(980)$ using $F_{K K}=\exp \left(-3 k_{K K}^{2}\right)$, where $k_{K K}$ is $K K$ momentum in $\mathrm{GeV} / \mathrm{c}$. Re $\Pi$ acts as an effective attraction. Parameters of $f_{0}(980)$ are known. Ref. [3] gives tables of pole positions when $M, G_{1}$ and $G_{2}$ are varied. If $M$ is as low as $500 \mathrm{MeV}$, there is still a pole at $806-i 78 \mathrm{MeV}$; for $M$ in the range $850-1100 \mathrm{MeV}$, there is a pole within $23 \mathrm{MeV}$ of the $K K$ threshold. The moral is that a strong threshold can move a resonance a surprisingly long way. For $f_{0}(980), G_{K K}^{2} \sim 0.7 \mathrm{GeV}^{2}$.

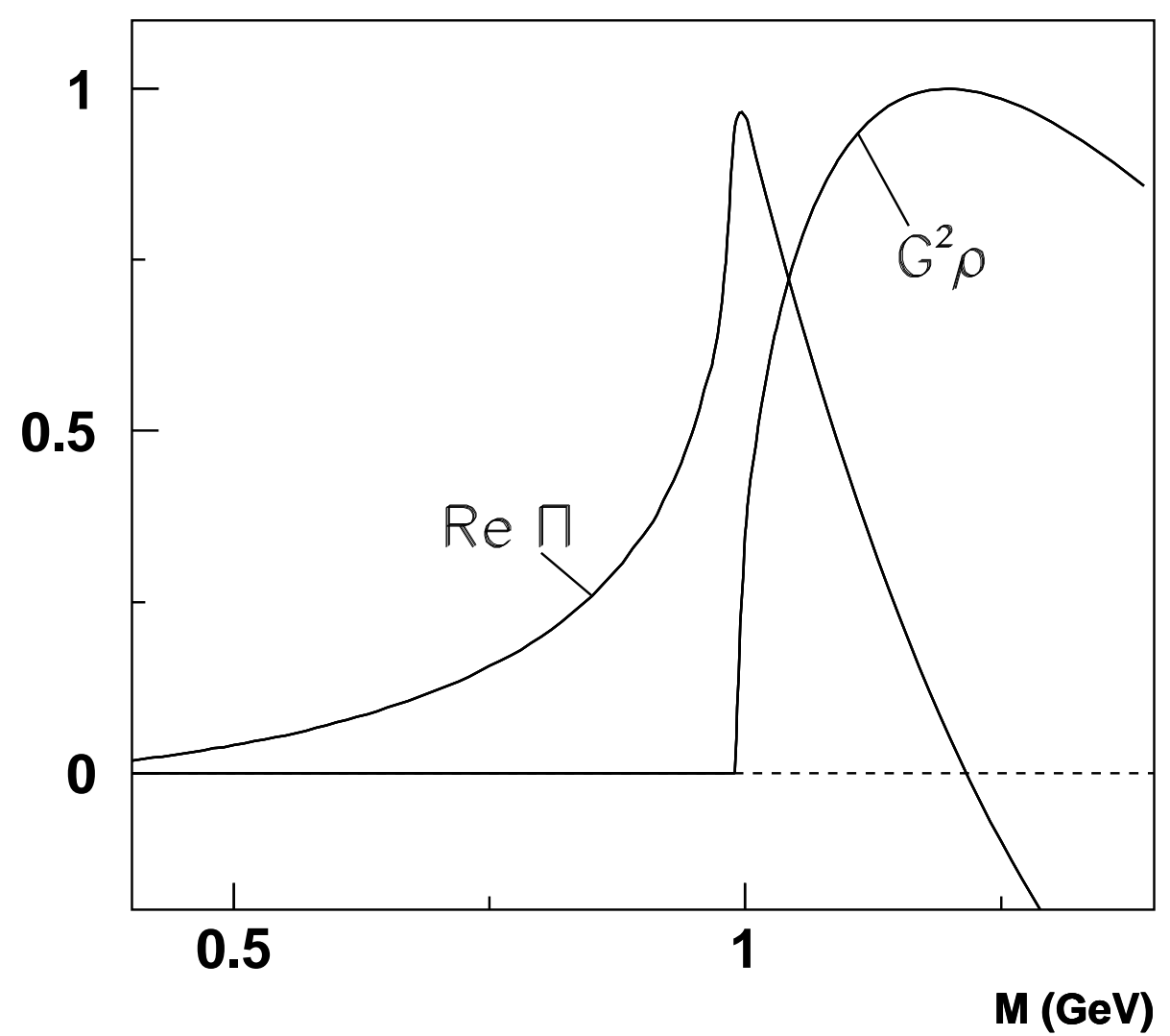

Figure 1: (a) $\operatorname{Re} \Pi_{K K}(s)$ and $G_{K K}^{2} \rho_{K K}(s)$ for $f_{0}(980)$, normalised to 1 at the peak of $G_{K K}^{2} \rho_{K K}$; (b) the loop diagram for $f_{0}(980) \rightarrow K \bar{K}$.

It is important to realise that $\operatorname{Re} \Pi$ is not an 'optional extra'. It is a rigorous consequence of analyticity for all $s$-channel decay processes. In principle these terms are required for all resonance decays. It is then logical to include also $t$ - and $u$-channel exchanges.

$\mathrm{P}$-wave thresholds lead to broader effects because of the $k^{3}$ momentum dependence of phase space. There are in principle contributions to $\rho(770)$ and $a_{1}(1260)$, but in practice these effects may be accomodated by fitted form factors describing decay widths.

The virtue of Eq. (2) is twofold. Firstly, it is easily evaluated, secondly it illustrates graphically the effect of the form factor $F$. Re $\Pi(s)$ goes negative close to the peak of $G^{2} \rho$ and subsequently has a minimum at $\sim 1.7 \mathrm{GeV}$; thereafter it slowly rises to 0 as $M \rightarrow \infty$.

There are alternatives to evaluating Eq. (2). The same result may be obtained by evaluating 
the loop diagram of Fig. 1(b). Secondly, solving the Bethe-Salpeter equation is equivalent to evaluating all loop diagrams. It is straightforward in principle to include $s$ - and $t$-channel exchanges in solving this equation.

Several authors have adopted a similar approach. Jaffe [5] gives the equations and discusses many of the implications in Section $2 \mathrm{C}$ of his paper. The Hamiltonian for a $q \bar{q}$ state decaying to meson-meson obeys

$$
H \Psi=\left(\begin{array}{cc}
H_{11} & V \\
V & H_{22}
\end{array}\right) \Psi ;
$$

$H_{11}$ describes short-range configurations; $H_{22}$ refers to ingoing and outgoing states and should include $t$ - and $u$-channel exchanges; $V$ accounts for the coupling between them due to $s$-channel decays.

Weinstein and Isgur pursued the connection between $q \bar{q}, q q \bar{q} \bar{q}$ and meson components in their work on $K \bar{K}$ molecules [6]. Van Beveren and Rupp [7] construct a model where the short-range attraction in $H_{11}$ is approximated by a harmonic oscillator potential, which couples at radius $R$ to ingoing and outgoing waves corresponding to decay channels. Despite the approximations, this gives valuable insight. Their algebraic solution satisfies the Schrödinger equation and is therefore fully analytic. It includes effects of thresholds fully, although not yet the $s$ - and $t$-channel exchanges.

Barnes and Swanson [8] consider meson loops due to pairs of $D, D^{*}, D_{s}$ and $D_{s}^{*}$ mesons, using the ${ }^{3} P_{0}$ model for decays. For $1 \mathrm{~S}, 1 \mathrm{P}$ and $2 \mathrm{P}$ charmonium states, they find that large mass shifts due to these loops may be 'hidden' in the valence quark model by a change of parameters. The important conclusion from their work is that two-meson continuum components of charmonium states may be quite large, with the result that the constituent quark model predicts masses which are too high, particularly near the thresholds of opening channels.

Oset and collaborators demonstrate in a series of papers that some resonances may be understood as 'dynamically driven states' 9] [10] [11] [12] [13] [14 [15] [16] due to $s, t$ and $u$-channel exchanges. They take the view that $\bar{q} q$ and $q q q$ components are not needed at all in these cases. This takes us full circle back to the approach tried by Chew.

How is it that Oset et al. are able to reproduce known resonances (approximately) with meson exchanges alone? They use S-wave form factors which are adjusted to get one predicted resonance of each paper at the right mass. Resulting amplitudes are strong. The form factors may be mocking up short range $q \bar{q}$ components. The importance of their work is the demonstration that components derived from meson loops are large, and should be taken into account.

On the other hand, the well known $J / \Psi,{ }^{3} P_{0}(3415),{ }^{3} P_{1}(3510),{ }^{3} P_{2}(3556), \Psi(2 S)$ and $\Psi(3770)$ are interpreted naturally as $c \bar{c}$ states (with tiny admixtures of the mesonic states to which they decay). Therefore it is logical to include the $c \bar{c}$ component into all other resonances unless there is a good reason why not. One should not be deterred from invoking $q \bar{q}$ and $q q q$ components to get all resonances with their correct masses and widths.

The central premise of the present paper is that both $H_{11}$ and $H_{22}$ play essential roles in all cases. This is different from approaches where only one of the two components in the Hamiltonian contributes, for example the approach based on four-quark mesonic states.

An approach which has recently been popular is to suppose that 'molecules' are formed from $\bar{q} \bar{q} q q$ configurations [17] [18][19] [20] [21]. The well known question over this approach is why so few tetraquarks are observed. Vijande et al. 222] throw light on this issue. They study the 
stability of pure $c \bar{c} n \bar{n}$ and $c c \bar{n} \bar{n}$ states in the absence of diquark interactions. They find that all $12 c \bar{c} n \bar{n}$ states with $J=0,1$ or 2 are unstable. Their calculation points to the conclusion that such molecules are rare unless either (i) there are attractive diquark interactions, or (ii) coupling to meson-meson final states contributes, as proposed here.

The layout of remaining sections is as follows. Section 2 considers a useful analogy with the covalent bond in chemistry. This analogy helps visualise the main features of mixing. Section 3 reviews approximations to be used in fitting data. Section 4 considers $\sigma, \kappa, a_{0}(980)$ and $f_{0}(980)$. Section 5 discusses firstly $X(3872)$ and concludes, as do several authors, that data require a linear combination of $c \bar{c}$ and $\bar{D} D^{*}$. Next it reviews the status of light mesons with $J^{P}=1^{-}, 2^{+}$and $0^{+}$. The objective is to demonstrate that observed states lie close to straight line trajectories when masses squared are plotted against radial excitation number. There are some significant deviations attributable to thresholds. However, the overall picture is consistent with rather regularly spaced $q \bar{q}$ states consistent with Regge trajectories. Such trajectories are usually attributed to flux tubes joining $q \bar{q}$ pairs and expanding as spin $J$ increases. There is no obvious reason why dynamically generated states should follow such a regular sequence. Section 6 makes remarks on desirable experiments and Section 7 draws conclusions.

\section{A helpful analogy with the covalent bond in chemistry}

The wave function $\Psi$ of Eq. (3) is a linear combination of $q \bar{q}$ (or $q q q$ ) and unconfined MM (or MB). The key point is that two attractive components $H_{11}$ and $H_{22}$ lower the eigenstate via the mixing. This is a purely quantum mechanical effect. There is a direct analogy with the covalent bond in chemistry. The solution of Eq. (3) is given by the Breit-Rabi equation:

$$
E=\left(E_{1}+E_{2}\right) / 2 \pm \sqrt{\left(E_{1}-E_{2}\right)^{2}+|V|^{2}}
$$

where $E_{1}$ and $E_{2}$ are eigenvalues of separate $H_{11}$ and $H_{22}$. One linear combination is pulled down in energy.

In chemistry, $H$ is in principle known exactly. The discussion of the hydrogen molecule (and more complex ones) is given in many textbooks on Physical Chemistry, for example the one of Atkins [23]. Consider two hydrogen atoms labelled $\mathrm{A}$ and $\mathrm{B}$, combining to make a hydrogen molecule; $H_{11}$ and $H_{22}$ describe $\mathrm{A}$ and $\mathrm{B}$. The equation describing this pair is precisely the same as Eq. (3). except that $H_{11}$ and $H_{22}$ have a different radial dependence to the mesonic case. Fig. 2(a) sketches contours of electron density for the lower state of Eq. (4). The effect of the mixing is that the electron density adjusts so that the two electrons are somewhat concentrated between the two ions. In more detail, the wave-function for the atomic molecule is expanded in terms of a complete set of atomic $\mathrm{H}$ orbitals. For hydrogen, these are just the energy levels of a single hydrogen atom. For a carbon atom, they are replaced by wave functions allowing for shielding of the Coulomb field of the nucleus by electrons in the lowest atomic levels. It is also necessary to anti-symmetrise wave functions. Atkin gives algebraic forms which approximate the electronic wave functions. With modern computing techniques, the Variational method adjusts the coefficients of the expansion in terms of excited states to describe wave functions with great accuracy.

Two key points are that (a) the extension of the wave function into the overlap region lowers momenta of electrons, hence their kinetic energy, (b) the whole system shrinks slightly and the 

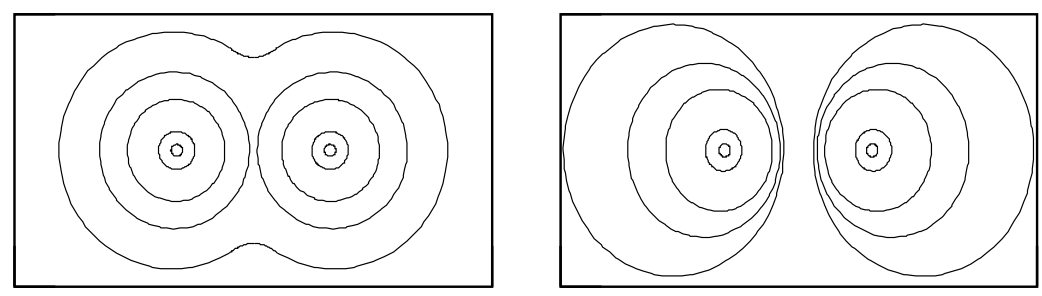

Figure 2: Sketch of the electron density in the hydrogen molecule for (a) lower and (b) upper states of Eq. (4).

binding of electrons to both nuclei increases.

In the Particle Physics case, the procedure is conceptually identical. The mesonic wave function for the ground state is sucked into the region of overlap, producing an attractive interaction between $q \bar{q}$ and meson-meson. This implies that $q \bar{q}$ will decay to final states where the interaction is indeed attractive. Fig. 2(b) shows the electron density for the upper state of Eq. (4). In this case, electrons are repelled from the overlap region, increasing their momenta and kinetic energies, hence the energy eigenvalue.

The second effect is that the increased binding for the lower state draws the quarks slightly down the Coulombic part of the QCD potential, shrinking the radius of the state. The converse happens for the upper state. Calculation of the mixing requires the radial wave functions of $q \bar{q}$ states, which can be evaluated from the funnel potential. A difficulty, however, is that the radial form of the mixing element $V$ between $q \bar{q}$ and $M M$ parts of the wave function is unknown, and has to be guessed. The calculations of Oset et al. concern purely the mesonic part of the wave function.

The conceptual analogy is simple: mixing between $q \bar{q}$ and meson-meson will lower the eigenvalue of the lower of the two states given by (4). The Variational Principle governs this eigenvalue and the wave function. The numerator of Eq. (2) is positive definite. It is therefore unavoidable that $\operatorname{Re} \Pi(s)$ goes negative at large $s$. The Variational Principle will cut off the high mass tail of the resonance, so as to minimise the repulsive part of $\operatorname{Re} \Pi(s)$, hence reducing the resonance width. A feature of light $q \bar{q}$ and $q q q$ resonances is that their widths are roughly equal to the spacing between radial excitations. It is likely that widths are restricted to this value by a feedback mechanism which creates an orderly sequence of resonances. A general feature of decays of high mass resonances is that S-wave decays to low mass final states are weak. Decays tend to be to high mass configurations with small momenta. High spin states generally decay with large angular momenta, where the centrifugal barrier delays the opening of the threshold.

There is little evidence for decays to $I=2 \pi \pi$ pairs or $I=3 / 2 K \pi$, where interactions are repulsive [24]. The commonly observed SU(3) octets and decuplets are those whose decays do not lead to such repulsive final states. Higher representions such as 27, 10 and $\overline{\mathbf{1 0}}$ do lead to such repulsive final states. The natural interpretation is that repulsive final states actively inhibit formation of representations higher than octets and decuplets. The Variational Principle arranges that the configurations produced are those where repulsive final states are suppressed. 


\section{Approximations}

A difficulty at present is that the form factor used in Fig. 1 is not known precisely. The usual Flatté formula, Eq. (1), serves as an approximate fitting function where $M$ and $g^{2}$ are fitted empirically. However, the cusp changes slope abruptly at the $K K$ threshold. Analysis of data then requires a precise knowledge of experimental resolution if the cusp is included in the fit. This is illustrated for $a_{0}$ (980) in Ref. [25], where Crystal Barrel data on $\bar{p} p \rightarrow \eta \pi^{0} \pi^{0}$ are fitted including the cusp. The mass resolution, $9.5 \mathrm{MeV}$, is known accurately in this case, but is large enough to smear out the cusp seriously. The cusp plays a strong role if the energy resolution is as good as $1 \mathrm{MeV}$. A further detail is that separate thresholds for $K^{+} K^{-}$and $K^{0} \bar{K}^{0}$ have not been used in Fig. 1 for simplicity; these two thresholds may be taken into account straightforwardly and the equations are given by Achasov and Shestakov [26].

\subsection{Broad Thresholds}

Although broad thresholds may play a role in forming a resonance, the dispersive term $\operatorname{Re} \Pi$ eventually has only a small effect on the experimental line-shape in most cases. There is, however, a crucial detail which has often been neglected in fits to data.

Let us consider as an example $\pi \pi \rightarrow \rho \rho$. The Breit-Wigner amplitude in this case is

$$
f=\frac{1}{k} \frac{M \sqrt{\Gamma_{\pi \pi}(s)} \sqrt{\Gamma_{4 \pi}(s)}}{M^{2}-s-\operatorname{Re} \Pi(s)-i M \Gamma_{\text {total }}(s)} .
$$

Here, $k$ is the $\pi \pi$ centre of mass momentum (allowing for the incident flux). The $\pi \pi$ phase space is approximately constant. However, it is essential to allow for the rapid $s$-dependence of $\Gamma_{4 \pi}$. Many experimental analyses ignore this point and fit $|f|^{2}$ to a Breit-Wigner resonance of constant width.

This is a critical point for many resonances in the mass range $1-2 \mathrm{GeV}$, where thresholds are opening. Fig. 3(a) shows line-shapes of $f_{0}(1370) \rightarrow \pi \pi$ and $4 \pi$ as an example. There is a large difference between them. This is the source of the large spread in masses reported by the Particle Data Group (PDG) for $f_{0}(1370)$ [27]. Anisovich et al. 28] determine the K-matrix pole position $1306 \pm 20 \mathrm{MeV}$ from a combined analysis of data on $\pi^{-} p \rightarrow \pi^{0} \pi^{0} n$ and $K \bar{K} n, \pi^{+} \pi^{-} \rightarrow \pi^{+} \pi^{-}$, and Crystal Barrel data for $\bar{p} p$ at rest $\rightarrow 3 \pi^{0}, \pi^{0} \eta \eta, \pi^{+} \pi^{-} \pi^{0}, K^{+} K^{-} \pi^{0}, K_{S}^{0} K_{S}^{0} \pi^{0}, K^{+} K_{S}^{0} \pi^{-}$ and $\bar{p} n \rightarrow \pi^{-} \pi^{-} \pi^{+}, K_{S}^{0} K^{-} \pi^{0}$ and $K_{S}^{0} K_{S}^{0} \pi^{-}$; the last three determine P-state annihilation. This analysis did not explicitly allow for $\operatorname{Re} \Pi(s)$ in the denominator. That was taken into account in Ref. [29], resulting in a mass of $1309 \pm 15 \mathrm{MeV}$, in close agreement with Anisovich et al. The peak in $4 \pi$, if judged from the mean of half-heights, is $1377 \mathrm{MeV}$, in good agreement with the determination of mass from $4 \pi$ data quoted by the PDG.

The Argand diagram from Ref. [29] is shown in Fig. 3(b). It follows a circle closely. The conclusion is that experimentalists can safely omit Re $\Pi$ as a first approximation. However, phase shifts depart significantly from a Breit-Wigner amplitude of constant width. At low mass, where $4 \pi$ phase space is small, $\Gamma_{\text {total }}$ is small and the phase shift varies rapidly; at high mass it varies more slowly. For high quality data, a second pass including $\operatorname{Re} \Pi(s)$ is desirable

Likewise $\eta(1405)$ and $\eta(1475)$ may be fitted as two decay modes of a single $\eta(1440)$ [30]. The $\eta(1475)$ is seen only in $K K^{*}(890)$, where phase space rises from threshold near $1385 \mathrm{MeV}$ as 

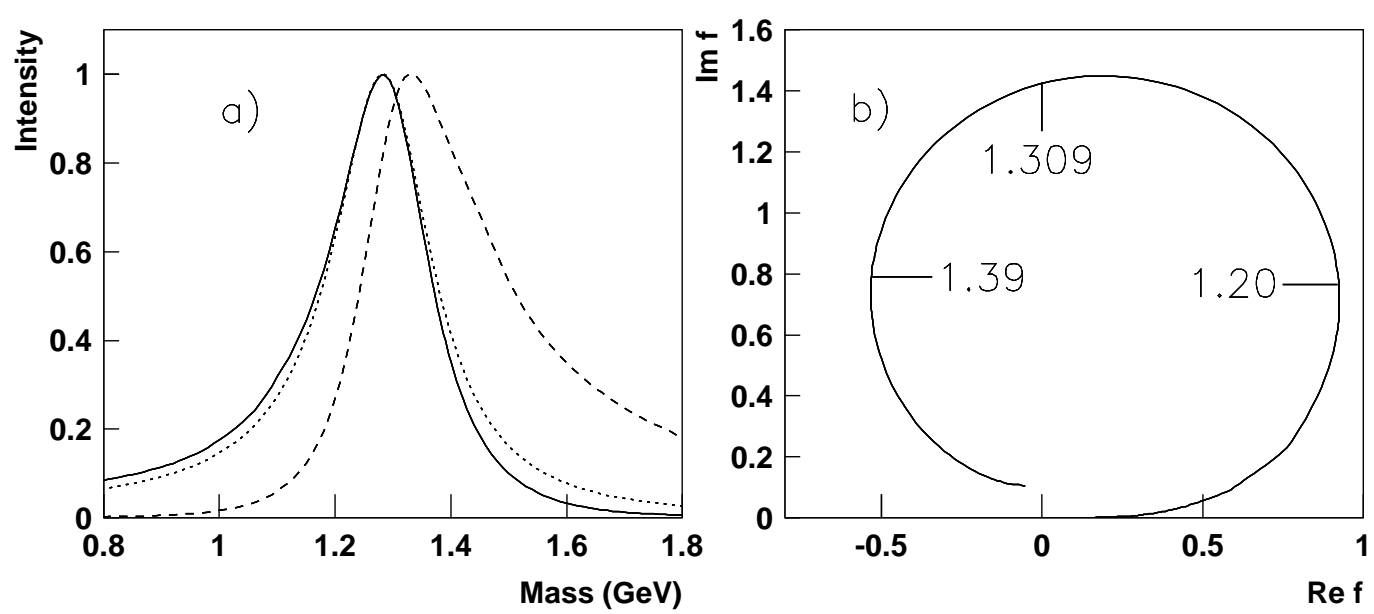

Figure 3: (a) Line-shapes of $f_{0}(1370)$ for $\pi \pi \rightarrow \pi \pi$ (full), a Breit-Wigner amplitude of constant width (dotted), and $\pi \pi \rightarrow 4 \pi$ (dashed); (b) the Argand diagram, with masses marked in GeV.

momentum cubed in the final state; the $\eta(1405)$ is seen in $\kappa K$ S-waves and $\eta \pi \pi$ where phase space changes slowly.

\section{$4 \sigma, \kappa, a_{0}(980)$ and $f_{0}(980)$}

The $\sigma$ and $\kappa$ poles are well predicted in both mass and width by the Roy equations [31] 32, which are based on $t$ - and $u$-channel exchanges. Exchange of $\rho(770)$ and $K^{*}(890)$ make strong contributions. The Julich group of Janssen et al. 33 showed that meson exchanges account for $f_{0}(980)$ and $a_{0}(980)$. It seems unavoidable that all four states $\sigma, \kappa, a_{0}(980)$ and $f_{0}(980)$ are strongly driven by meson exchanges. They are conspicuous because $q \bar{q}$ states lie in the mass range 1300-1700 MeV. There is one feature, however, which is hidden in the meson exchanges going into the Roy equations. They impose the Adler zero coming from chiral symmetry breaking. This is a short-range effect.

Jaffe has proposed [34] that $\sigma, \kappa, a_{0}(980)$ and $f_{0}(980)$ are colourless 4-quark states made from a coloured SU(3) $\mathbf{3}$ combination of $q q$ and a $\overline{\mathbf{3}}$ combination of $\bar{q} \bar{q}$. This naturally leads to a light $\sigma$, an intermediate $\kappa$ and the highest (degenerate) masses for $a_{0}(980)$ and $f_{0}(980)$, in agreement with experiment. Note, however that meson-meson configurations lead to a similar spectrum except that the $a_{0}(980)$ might lie at the $\eta \pi$ threshold. This does not happen because of the nearby Adler zero at $s=m_{\eta}^{2}-m_{\pi}^{2} / 2$; the $a_{0}$ (980) migrates to the $K K$ threshold because the Adler zero in this case is distant, at $s=m_{K}^{2} / 2$ [35]. Jaffe's model does not agree well with the observed decay branching ratio $(\sigma \rightarrow K K) /(\sigma \rightarrow \pi \pi)$ near $1 \mathrm{GeV}$. [36] A serious problem is that, from the width of the $\sigma$ pole, the $\kappa$ width is predicted to be $(236 \pm 39) \mathrm{MeV}$, much less than the latest value: $758 \pm 10$ (stat) \pm 44 (syst) $\mathrm{MeV}$ [24].

A further point is that Maiani et al. [17] extend Jaffe's scheme to $[c q][\bar{q} q]$ configurations $\mathbf{6} \otimes \overline{\mathbf{3}}$. They give a firm prediction for the observation of analogues of $a_{0}(980)$ in $c \bar{s} n \bar{n}$ with $I=1$ and charges $0,+1$ and +2 . There is no evidence for such states as yet. 
Although experimental line-shapes are not affected strongly for broad resonances, one should not jump to the conclusion that broad resonances are pure $q \bar{q}$ states. The calculations of Oset et al. show that mesonic components are potentially large. A related point is what happens to the upper energy combinations appearing in Eq. (4). If mesonic components are large, these upper combinations are moved upwards substantially. As Jaffe remarks, they become broad and are likely to fall apart, creating a broad high mass background. The high mass tail of the $\sigma$ does behave in this sort of way above $1 \mathrm{GeV}$, due to coupling to $4 \pi$ [29]; however, the precise form of this high mass behaviour is poorly known because of lack of data on $\pi \pi \rightarrow 4 \pi$.

\section{Applications}

\section{$5.1 \quad X(3872)$}

It is evident from the width of the cusp in $R e \Pi$ of Fig. 1 that a cusp alone fails to fit the $\sim 3$ $\mathrm{MeV}$ width of $X(3872)$ [27]; the coupling to $\bar{D} D^{*}$ is weaker than that of $f_{0}(980)$ to $K K$, but the shape of the dispersion curve is similar.

Several authors conclude that a linear combination of $c \bar{c}$ and $\bar{D} D^{*}$ is likely in $X(3872)$. Eichten et al. 37 remark that this explains the low mass of $X(3872)$ compared to early calculations based purely on $c \bar{c}$. Suzuki [38] points out that the large production rate of $X(3872)$ in CDF data from the Tevatron [39] requires that it has a large wave function at the origin, hence a substantial $c \bar{c}$ component; for a pure molecular state the observed production rate is nearly 2 orders of magnitude smaller than CDF observe. Conversely, the molecular re-arrangements $\bar{D} D^{*} \rightarrow \rho J / \Psi$ and $\omega J / \Psi$ account naturally for the weak decays which are observed; here, the strength of $\bar{D} D^{*}$ binding is unimportant. Bignamini et al. confirm that the CDF production rate is about 2 orders of magnitude too large to be explained by a molecular component alone [40]. Swanson [41] also favoures mixing between $\bar{c} c$ and a molecular component.

Recently Lee et al. [42] have made a detailed fit to existing data, solving the Bethe-Salpeter equation - equivalent to evaluating the dispersion integral of Eq. (2). The binding energy of $X(3872)$ arises essentially from $\bar{D} D^{*}$ loop diagrams. The magnitude of $\pi$ exchange is known from the width of the decay $D^{*} \rightarrow D \pi$; other exchanges are modelled. However, meson exchanges are not the essential source of binding. They simply need to be attractive, so that $\bar{D}$ and $D^{*}$ approach one another. Both $\bar{D}_{0} D_{0}^{*}$ and $D^{+} D^{-}$channels contribute, though the $X(3872)$ appears at the lower threshold. The binding energy is controled sensitively by the form factor.

Kalashnikova and Nefediev conclude that $X(3872)$ has substantial components of both $\bar{c} c$ and $\bar{D} D^{*}$ [43]. They point out that Babar data for the ratio of branching ratios $B R[X(3872) \rightarrow$ $\left.\gamma \Psi^{\prime}(2 S)\right] / B R[X(3872) \rightarrow \gamma J / \Psi]=3.4 \pm 1.4$ [44] agrees within a factor 2 with estimates for a $\bar{c} c$ state [45]. The prediction of Swanson for a pure molecule is $\simeq 4 \times 10^{-3}$ [46]. Also Gutsche et al. conclude that some component of $c \bar{c}$ is essential to explain the large rate for $X(3872) \rightarrow \gamma \Psi^{\prime}$ [47]. So there seems to be widespread agreement that $X(3872)$ is a linear combination of $c \bar{c}$ and $D \bar{D}^{*}$. The precise combination is not yet agreed.

The narrow width of $X(3872)$ arises because its decay modes to $\pi^{+} \pi^{-} J / \Psi, \omega J / \Psi$ and possibly $\chi(3510) \sigma$ are OZI suppressed, therefore weak. Its coupling to $\bar{D}^{0} D^{* 0}$ over the width of the resonance is also weak. However, the coupling to $\bar{D} D^{*}$ rises rapidly above threshold and produces the binding via virtual loop diagrams. Kalashnikova and Nefediev conclude that the $\bar{c} c$ state 
is attracted to the $\bar{D} D^{*}$ threshold. Ortega et al. [48 reach a similar conclusion that $X(3872)$ must have a large $\bar{D} D^{*}$ component.

\subsection{Not all cusps are resonances}

There is a cusp at the $\pi d$ threshold [49], but no resonance. The exotic $Z^{+}(4430)$ of Belle [50] is at the threshold for $D^{*}(2007) D_{1}(2420)$ and has a width close to that of $D_{1}(2420)$. The data can be fitted as a resonance, but can also be fitted successfully by a non-resonant cusp, see Fig. 6 of [3]. Additionally, Babar do not confirm the existence of the $Z(4430)$.

\subsection{Light Vector Mesons}

Crystal Barrel data in flight, taken together with other data at lower energies, provide evidence that resonance masses squared for each spin-parity lie close to straight line trajectories [51]. Updated examples are shown here in Fig. 3. They resemble Regge trajectories, except they are drawn for one set of quantum numbers at a time. There is a striking agreement for all $J^{P}$, with a common slope of $1.143 \pm 0.013 \mathrm{GeV}^{2}$ for each unit of excitation. A similar regularity is observed for baryon resonances with similar slope [52]. Such regularity agrees with states having a large $q \bar{q}$ component, but there is no direct connection with molecules or dynamically generated states.

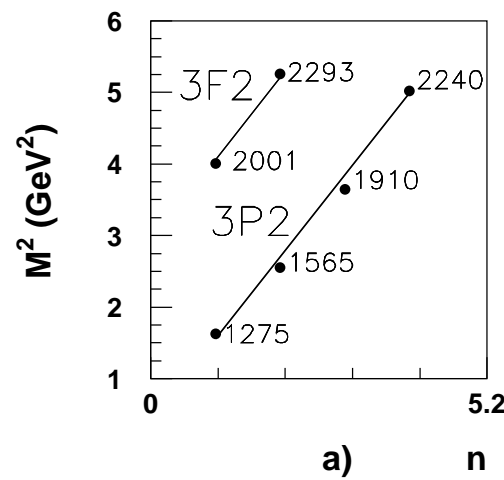

Figure 4: Trajectories of $\bar{n} n$ resonances: (a) $I=1, J^{P C}=1^{--}$, (b) $I=0, J^{P C}=2^{++}$, (c) $I=0, J^{P C}=4^{++}$and $0^{++}$.

An application of the idea that some resonances mix strongly with channels to which they decay concerns $\rho(1900)$. This state lies close to the $N \bar{N}$ threshold and it is well known that the $\bar{p} p^{3} S_{1}$ interaction is strongly attractive. Babar and E687 observe it in decays to $3 \pi^{+} 3 \pi^{-}$and $2\left(\pi^{+} \pi^{-} \pi^{0}\right)$ [27]. These are strong decay modes in $N \bar{N}$ annihilation. It is natural to interpret $\rho(1900)$ as the $n=3{ }^{3} S_{1} n \bar{n}$ state mixed with $\bar{p} p$. Then other $\rho$ states fall into place as follows: (ii) $\rho(2000)={ }^{3} D_{1}, n=2$. It is observed in three sets of data: $\pi^{+} \pi^{-}, \pi \omega$ and $a_{0} \omega$. There are extensive differential cross section and polarisation data on $\bar{p} p \rightarrow \pi^{+} \pi^{-}$from the PS 172 experiment down to a mass of $1910 \mathrm{MeV}$ (a beam momentum of $360 \mathrm{MeV} / \mathrm{c}$ ) [53]. There are further similar data above a beam momentum of $1 \mathrm{GeV} / \mathrm{c}$ from an experiment at the Cern PS of Eisenhandler et al [54]. The polarisation data determine the ratio of decay amplitudes to 
${ }^{3} D_{1}$ and ${ }^{3} S_{1} \bar{p} p$ configurations: $r_{D / S}=g_{\bar{p} p}\left({ }^{3} D_{1}\right) / g_{\bar{p} p}\left({ }^{3} S_{1}\right)=0.70 \pm 0.32 ;$ for the low available momentum in $\bar{p} p$, this is a rather large ${ }^{3} D_{1}$ component.

(iii) $\rho(2150)={ }^{3} S_{1}, n=4$. It is seen in $\pi^{+} \pi^{-}$data of [53] and [54] and in Crystal Barrel data for $a_{0}(980) \pi$ and in GAMS and Babar data [27]; (the PDG incorrectly lists the $1988 \mathrm{MeV}$ state of Hasan [55] under $\rho(2150)$, but it is the $\rho(2000))$. For $\rho(2150), r_{D / S}=-0.05 \pm 0.42$.

(iv) $\rho(2265)={ }^{3} D_{1}, n=3$. It is observed only in two sets of data, $\pi^{+} \pi^{-}$and in Crystal Barrel data for $a_{2} \omega$ and therefore needs confirmation; it has a large error for $r_{D / S}$. The $\rho(1700), \rho(2000)$ and $\rho(2265)$ are consistent within errors with a straight trajectory with the same slope as other states, see Fig. 2(b).

(v) The $Y(2175)$ [27] observed by BES 2 and Babar in $\phi f_{0}(980)$ and $K^{+} K^{-} f_{0}(980)$ makes a natural $s \bar{s}$ partner for $\rho(2000)$. Note that there is sufficient momentum in the final state to allow a ${ }^{3} D_{1}$ state.

\section{$5.4 J^{P}=2^{+}$light mesons}

The $f_{2}(1565)$ lies at the $\omega \omega$ threshold. The PDG quotes an average mass of $1562 \pm 13 \mathrm{MeV}$, although Baker et al. [56] find a mass of $1598 \pm 11$ (stat) \pm 9 (syst) $\mathrm{MeV}$ when $\operatorname{Re} \Pi(s)$ is included in the analysis. This is distinctly lower than the mass of $a_{2}(1700)$, in the range 1660-1732 MeV. For almost massless quarks, one expects $f_{2}$ and $a_{2}$ masses to be close. So $f_{2}(1565)$ has clearly been attracted to the $\omega \omega$ threshold. This requires a molecular component. The $f_{2}(1565)$ appears clearly in $\pi \pi$, as observed by several groups [27]. It should appear in $\rho \rho$ with $g_{\rho \rho}^{2}=3 g_{\omega \omega}^{2}$ by $\mathrm{SU}(2)$ symmetry, which predicts $g\left(\rho^{0} \rho^{0}\right)=-g(\omega \omega)$ because of the similar masses of light quarks and the close masses of $\rho(770)$ and $\omega(782)$.

The $f_{2}(1640) \rightarrow \omega \omega$ observed by GAMS and VES [27] may be fitted by folding the line-shape of $f_{2}(1565)$ with $\omega \omega$ phase space and a reasonable form factor [56], together with the dispersive term $\operatorname{Re} \Pi(s)$. There is no need for separate $f_{2}(1565)$ and $f_{2}(1640)$; this has confused a number of theoretical predictions of the sequence of $2^{+}$states.

Fig. 2(b) shows trajectories for $2^{++}$states, including those above the $\bar{p} p$ threshold from Crystal Barrel data in flight, using trajectories with a slope of $1.14 \mathrm{GeV}^{2}$. The PDG makes a number of serious errors in reporting the Crystal Barrel publications. Those mistakes distort conclusions to be drawn from the data. It lists $f_{2}(2240)$ under $f_{2}(2300)$, which is observed in $\phi \phi$ and $K K$ by all other groups. The $f_{2}(2300)$ is naturally interpreted as an $s \bar{s}$ state. Both $f_{2}(2240)$ and $f_{2}(2293)$ are observed in a combined analysis of ten sets of data: four sets of PS172 and Eisenhandler et al., together with Crystal Barrel data for $\eta \pi^{0} \pi^{0}, \eta^{\prime} \pi^{0} \pi^{0}, \eta \eta \eta, \pi^{0} \pi^{0}, \eta \eta$ and $\eta \eta^{\prime}$. The data from the last 3 channels are fitted to a linear combination $\cos \phi|n \bar{n}>+\sin \phi| s \bar{s}>$ and the mixing angle is determined to be $\phi=7.5^{\circ}$ for $f_{0}(2240)$ and $\phi=-14.8^{\circ}$ for $f_{2}(2293)$ [57]. So the $f_{2}(2240)$ is certainly not an $s \bar{s}$ state. From polarisation data, the $f_{2}(2240)$ is dominantly ${ }^{3} P_{2}$ with $r_{F / P}=0.46 \pm 0.09$ (defined like $\left.r_{D / S}\right)$ and the $f_{2}(2300)$ is largely ${ }^{3} F_{2}$ with $r_{F / P}=-2.2 \pm 0.6$. The PDG fails to list the $f_{2}(2293)$ at all, despite many prompts over a 6 year period. It is observed in 5 channels: $\pi \pi, \eta \eta, \eta \eta^{\prime}, f_{2} \eta$ and $a_{2}(1320) \pi$.

A further comment is that the $f_{2}(2150)$ is conspicuous by its absence from Crystal Barrel data in flight. All $s \bar{s}$ states such as $f_{2}(1525)$ are produced very weakly in $\bar{p} p$ interactions. The $f_{2}(2150)$ is observed mostly in $K \bar{K}$ and $\eta \eta$ channels. It is therefore naturally interpreted as an $s \bar{s}$ state, the partner of $f_{2}(1905)$.

An important systematic observation is that $\bar{p} p$ states tend to decay with the same $L$ as the 
initial $\bar{p} p$ state. There is a simple explanation, namely good overlap of the initial and final states in impact parameter. This observation may be useful to those calculating decays, hence mesonic contributions to eqns. (1)-(4).

\subsection{Light $0^{+}$mesons}

There is extensive evidence for a radial excitation of $f_{0}(1370)$ at $1790 \mathrm{MeV}$. The facts run as follows. In BES 2 data for $J / \Psi \rightarrow \omega K^{+} K^{-}$, there is a clear $f_{0}(1710) \rightarrow K K$ [58. In high statistics data for $J / \Psi \rightarrow \omega \pi^{+} \pi^{-}$, [59] there is no visible $f_{0}(1710)$, setting a limit on branching ratios: $B R\left(f_{0}(1710) \rightarrow \pi \pi\right) / B R\left(f_{0}(1710) \rightarrow K K\right)<0.11$ with $95 \%$ confidence. Thirdly, in $J / \Psi \rightarrow \phi \pi^{+} \pi^{-}$, there is a $\pi \pi$ peak requiring an additional $f_{0}(1790)$ decaying to $\pi \pi$ but weakly to $K K$ [60]. There is ample independent evidence for it in $J / \Psi \rightarrow \gamma 4 \pi$ [61] [62] 63] and $\bar{p} p \rightarrow \eta \eta \pi^{0}$ in flight [64]. BES 2 also report an $\omega \phi$ peak of 95 events at $1812 \mathrm{MeV} ; J^{P}=0^{+}$ is favoured [65]. It is confirmed by VES data at the Hadron09 conference [66]. The BES data may be fitted well with the $f_{0}(1790)$ line-shape folded with $\omega \phi$ phase space and a form factor $\exp -3 k_{\omega \phi}^{2}$. There is some scatter on Fig. 4(c) of masses about the line of standard slope; this may well be because masses of $0^{+}$states tend to be the most difficult to deterine due to isotropic angular distributions.

A comment is needed on $f_{2}(1810)$ of the Particle Data Tables. It does not fit in naturally in Fig. 2(b). The spin analysis of the GAMS group [67] finds a very marginal difference between spin 0 and spin 2. It rests on a fine distinction in the angular distribution depending strongly on experimental acceptance; however, no Monte Carlo of the acceptance is shown. With the benefit of hindsight, it seems possible that this was in fact the first observation of $f_{0}(1790)$.

Summarising subsections 5.3 to 5.5, there is strong evidence in Figs. 4(a)-(c) that resonances lie close to straight trajectories as a function of mass squared. These may be redrawn as Regge trajectories for $1^{-}, 2^{+}, 3^{-}$, etc., see Fig. 56 of [51]. Regge trajectories are naturally explained by a flux tube joining $q$ and $\bar{q}$; the energy stored in the flux tube explains the linear relation between $J$ and mass squared. There is no clear reason why molecules or dynamically generated states should follow such trajectories.

\subsection{Glueballs}

Morningstar and Peardon [68] predict glueball masses in the quenched approximation where $q \bar{q}$ are omitted. When mixing with $q \bar{q}$ is included, mixing is likely to lower glueball masses.

\section{$6 \quad$ Remarks on further experiments}

Further progress towards a complete spectroscopy of light mesons and baryons is important for an understanding of confinement - one of the key phase transitions in physics. Progress is possible by measuring transverse polarisation in formation processes. Consider $\bar{p} p$ as an example. The high spin states appear clearly as peaks, e.g. $f_{4}(2050)$ and $f_{4}(2300)$. These serve as interferometers for lower states. However, differential cross sections measure only real parts of interferences. This leaves the door open to two-fold ambiguities in relative phases and large errors if resonances happen to be orthogonal. A measurement of transverse polarisation normal 
to the plane of scattering measures $\operatorname{Tr}<A^{*} \sigma_{y} A>$, where $A$ is the amplitude. This measures the imaginary part of interferences. What appears to be less well known is that transverse polarisation in the plane of scattering gives additional information for three and four-body final states with a decay plane different to the plane defined by the beam and initial state polarisation. This depends on $\operatorname{Tr}<A^{*} \sigma_{x} A>$, and measures the real parts of exactly the same interferences as appear from the $\sigma_{y}$ operator. Longitudinal polarisation depends only on differences of two intensities and is less useful.

An example of a simple experiment which would pay a rich dividend is to measure such polarisations with the Crystal Barrel detector at the forthcoming GSI $\bar{p}$ source, over the same mass range as used at LEAR. An extracted beam with these momenta will be available at the FLAIR ring. Such measurements could indeed have been made at LEAR if it had not been sacrificed to the funding of the LHC. The present situation is that the amplitudes for $I=0$, $C=+1$ states are unique for all expected $J^{P}$. For $I=1, C=-1$, they are nearly complete, but there are some weaknesses for low spin states, notably ${ }^{3} S_{1}$ which leads to a flat decay angular distribution. For $I=1, C=+1$ there is a two-fold ambiguity for $\eta \pi$ final states and crucial $J^{P}=0^{+}$states are missing. For $I=0, C=-1$ there are many missing states.

The measurements required are to (i) $\eta \pi$ and $\eta \eta \pi(I=1, C=+1)$, (ii) $\omega \pi(I=1, C=-1)$, (iii) $\omega \eta$ and $\omega \eta \pi^{0}(I=0, C=-1)$. A measurement of $\eta \pi^{0} \pi^{0}$ would cross-check the existing solution and provide information on interferences between singlet and triplet $\bar{p} p$ states. As well as locating missing states, this would build up a clear picture of the many decays observable, for comparision with meson exchange processes. All of these channels can be measured simultaneously with the existing Crystal Barrel detector.

A Monte Carlo simulation of results extrapolated from existing analyses predicts a unique set of amplitudes for all quantum numbers. Data are required from $2 \mathrm{GeV} / \mathrm{c}$ down to the lowest possible momentum $\sim 360 \mathrm{MeV} / \mathrm{c}$. Seven of the nine momenta studied at LEAR were run in 3 months of beam time, so it is not a long experiment, nor does it demand beam intensities above $5 \times 10^{4} \bar{p} / s$. A Monte Carlo study shows that backgrounds from heavy nuclei in the polarised target (and its cryostat) should be at or below an average level of 10\%; this is comparable with cross-talk between final states and is easily measured from a dummy target.

Baryon spectrocopy would also benefit from similar $\pi^{ \pm} p$ polarisation measurements in inelastic channels. A transverse magnetic field is required for compatibility with the polarised target. Rates are enormous, so running time is governed by down-time required for polarising the target and changing momenta. Data at $30 \mathrm{MeV}$ steps of mass appear sufficient, except close to 2-body thresholds such as $\omega N$.

\section{Conclusions}

The objective of this paper has been to make a case for what appears logically necessary, namely that both quark combinations at short range and decay channels at large range contribute to the eigenstates. The $X(3872)$ is a prime example of mixing between $c \bar{c}$ and meson-meson in the form of $\bar{D} D^{*}$.

In view of the calculations of Oset et al. and Barnes and Swanson, it seems likely that many resonances contain large mesonic contributions. The straight trajectories of Figs. 2(a)-(c) are naturally explained by Regge phenomenology; molecules and dynamically generated states 
provide no obvious explanation of these trajectories. The large mass shift between $f_{2}(1565)$ and $a_{2}(1700)$ indicates meson mixing into $f_{2}(1565)$, lowering the eigenvalue in analogy with the covalent bond in chemistry.

The data on $a_{0}(980), f_{0}(980), f_{2}(1565)$ and $\rho(1900)$ fit naturally into this picture. There must be a large mesonic contribution to the nonet of $\sigma, \kappa, a_{0}(980)$ and $f_{0}(980)$, but there could be a modest diquark component as well. It is likely that there will be a small $q \bar{q}$ component, but this is suppressed by the $L=1$ centrifugal barrier for ${ }^{3} P_{0}$ combinations.

Experimentalists must take care to fit the $s$-dependence of the numerator of Breit-Wigner resonances due to phase space, e.g. $f_{0}(1370) \rightarrow 2 \pi$ has a very different line-shape to $f_{0}(1370) \rightarrow$ $4 \pi$. The denominator may be fitted as a first approximation with a Breit-Wigner resonances of constant width; however, for high quality data, the effect of the dispersive component in the real part of the denominator matters. For sharp thresholds, e.g. $f_{0} \rightarrow K K$, the Flatté formula is an approximation; with high quality data, the correction due to the cusp in $\operatorname{Re} \Pi(s)$ is important, but requires precise information on experimental resolution.

Further experiments on transverse polarisation in inelastic processes are needed and appear to be practicable without large cost.

\section{References}

[1] Chew G F (1966) "The analytic S-matrix: a basis for nuclear democracy" (Benjamin, New York)

[2] Donnachie A and Hamilton J (1965) Phys. Rev. 138 B 678

[3] Bugg D V (2008) J Phys. G: Nucl. Part. Phys. 35075005

[4] Flatté S M (1976) Phys. Lett. B 63224

[5] Jaffe R L (2007) Prog. Theor. Phys. Suppl. 168127

[6] Weinstein J and Isgur N (1990) Phys. Rev. D 412236

[7] van Beveren E and Rupp G (2001) Eur. Phys. J C 22493

[8] Barnes T and Swanson E S (2008) Phys. Rev. C 77055206

[9] Gamermann D, Oset E, Strottman D and Vicente Vacas M J (2007) Phys. Rev. D 76074016

[10] Geng L S, Oset E, Peláez J R and Roca L (2009) Eur. Phys. J A 3981

[11] Gonzalez P, Oset E and Vijande J (2009) Phys. Rev. C 79025207

[12] Geng L S and Oset E (2009) Phys. Rev. D 79074009

[13] Molina R and Oset E (2009), Phys. Rev. D 80114013

[14] Sarkar S, Sun B-X, Oset E and Vicente Vacas M J (2009) preprint arXiv: 0902.3150

[15] Molina R, Nagahiro H, Hosaka A and Oset E (2009) Phys. Rev. D 80014025 
[16] Oset E and Ramos A (2007) preprint arXiv: 0905.0973

[17] Maiani L, Piccinini F, Polosa A D and Riquer V (2004) Phys. Rev. Lett. 93212002

[18] Braaten E and Lu M (2008) Phys. Rev. D 77014029 and references given there

[19] Hanhart C, Guo F-K and Meißner U-G (2008) Phys. Lett B 66526

[20] Alvarez-Ruso L, Oller J A and Alarcón J M (2009) Phys. Rev. D 80054011

[21] Martinéz Torres A et al. 2008 Phys. Rev. D 78074031

[22] Vijande J, Valcarce A, Richard J-M and Barnea A (2009) Few Body Syst 4599

[23] Atkins P W (1983) "Molecular Quantum Mechanics", Oxford Univ. Press, Oxford, 2nd Edition, Chapter 10

[24] Bugg D V (2010) Phys. Rev. D 81014002

[25] Bugg D V (2008) Phys. Rev. D 78 074023, Fig. 6(b)

[26] Achasov N N and Shestakov G N (2009) preprint arXiv: 0910.4706. Fig. 6(a)

[27] Amsler C et al. (Particle Data Group) (2008) Phys. Lett. B 6671

[28] Anisovich V V al. (2003) Eur. Phys. J A 16229

[29] Bugg D V (2003) Eur. Phys. J C 5255

[30] Bugg D V (2009) preprint arXiv: 0907.3015

[31] Caprini I, Colangelo G and Leutwyler H (2006) Phys. Rev. Lett. 96132001

[32] Descotes-Genon S and Moussallam B (2006) Eur. Phys. J C 48553

[33] Janssen G, Pearce B C, Holinde K and Speth J (1995) Phys. Rev. D 522690

[34] Jaffe R L (1977) Phys. Rev. D 14 267, 281

[35] van Beveren E, Bugg D V, Kleefeld F and Rupp G (2006) Phys. Lett. B 641265

[36] Bugg D V (2006) Eur. Phys. J C 4757

[37] Eichten E J, Lane K and Quigg C (2006) Phys. Rev. D 73 014014; Erratum ibid (2006) D 73079903

[38] Suzuki M (2005) Phys. Rev. D 72114013

[39] Acosta D et al. (CDF Collaboration) (2004) Phys. Rev. Lett. 93072001

[40] Bignamini C et al. (2000) Phys. Rev. Lett. 103162001

[41] Swanson E S (2006) Phys. Rept. 429243 
[42] Lee I W, Faessler A, Gutsche T and Lyubovitskij V E (2009) Phys. Rev. D 80094005

[43] Kalashnikova Yu S and Nefediev A V (2009) Phys. Rev. D 80074004

[44] B. Aubert et al. (Babar Collaboration) (2009) Phys. Rev. Lett. 102132001

[45] Barnes T, Godfrey S and Swanson E S (2005) Phys. Rev. D 72054026

[46] Swanson E S (2004) Phys. Lett. B 598197

[47] Gutsche T et al. (2010) preprint arXiv: 1001.1870

[48] Ortega P G, Segovia J, Enten D R and Fernandez F (2009) preprint arXiv: 0907.3997

[49] Spuller J and Measday D F (1975) Phys. Rev. D 12 3550, Fig. 2

[50] Choi S K et al. (Belle Collaboration) (2008) Phys. Rev. Lett. 100142001

[51] Bugg D V (2004) Phys. Rep. 397257

[52] Klempt E (2002) Phys. Rev. C 66058201

[53] Hasan A et al. (1992) Nucl. Phys. B 3783

[54] Eisenhandler E et al. (1975) Nucl. Phys. B 98109

[55] Hasan A and Bugg D V (1994) Phys. Lett. B 334215

[56] Baker C A et al. (1999) Phys. Lett. B 4671147

[57] Anisovich A V et al. (1999) Nucl. Phys. A 662319

[58] Ablikim M et al. (BES 2 Collaboration) (2004) Phys. Lett. B 603138

[59] Ablikim M et al. (BES 2 Collaboration) (2004) Phys. Lett. B 598149

[60] Ablikim M et al. (BES 2 Collaboration) (2005) Phys. Lett. B 607243

[61] Bugg D V et al. (1995) Phys. Lett. B 353378

[62] Bai J Z et al. (BES 1 Collaboration) (2000) Phys. Lett. B 472207

[63] Bugg D V (2009) preprint arXiv: 0907.3021

[64] Anisovich A V et al. (1999) Phys. Lett. B 449154

[65] Ablikim M et al. (BES 2 Collaboration) (2006) Phys. Rev. Lett. B 96162002

[66] Ivashin A (2009) Hadron09 conference

[67] Alde D M et al. (1987) Phys. Lett. B 198286

[68] Morningstar C J and Peardon M (1999) Phys. Rev. D 60034509 